\title{
Crustal deformation associated with crustal activities in the northern Izu-islands area during the summer, 2000
}

\author{
Masaru Kaidzu, Takuya Nishimura, Makoto Murakami, Shinzaburo Ozawa, Takeshi Sagiya, Hiroshi Yarai, and Tetsuro Imakiire \\ Geographical Survey Institute, Kitasato-1, Tsukuba, Ibaraki 305-0811, Japan
}

(Received September 6, 2000; Revised September 8, 2000; Accepted September 8, 2000)

\begin{abstract}
In the end of June, 2000, intense crustal activity took place in Miyake-jima, Niijima, Kozu-shima and their vicinity. Here we report on the crustal deformation in the area during the period from June 24 to September 4, 2000, detected with the nationwide Global Positioning System (GPS) array operated by the Geographical Survey Institute. The deformation in this area during the above period is characterized by the deflation of Miyake-jima and the extension of the crust in the northeast-southwest direction over a wide area.
\end{abstract}

\section{Area of Interest and Overall Displacements of GPS Stations}

On June 26, 2000, earthquake swarm activity took place in Miyake-jima. The crustal deformation and the migration of epicenters of earthquakes suggested that magma intruded underneath the southwestern part of Miyake-jima and the magma migrated to the west. This event was followed by large deflation of Miyake-jima, intense earthquake swarm activity in the sea surrounded by Niijima, Kozu-shima and Miyake-jima, and rapid deformations in the area. Such crustal deformations have been detected with the nationwide GPS array named GPS Earth Observation Network (GEONET) (Miyazaki et al., 1998) and operated by the Geographical Survey Institute. The crustal activity still persists at the stage of September 4, 2000.

Figure 1 shows the area where the above-mentioned activity has been taking place. Solid squares with numbers denote GPS stations. Figure 2 shows the overall displacements of the GEONET GPS stations in the area and its vicinity. It is noteworthy that significant deformations could be detected as far north as the Izu-Oshima island, the Miura peninsula, the southern parts of the Izu and the Boso peninsulas. The pattern of arrows, especially the displacement of Shikinejima strongly suggests that the cause of deformation is the opening of a dike-like crack in the southeast of Shikine-jima.

\section{Temporal Variations in the Components of Base- line Vectors}

Figures 3 to 8 show the deformation of the Miyake-jima island during the period from 0:00 universal time (UT), June 24 to 6:00 UT, September 4, with a plotting interval of 3 hours. The GPS data were collected every 3 hours and the coordinates were estimated from the data covering the overlapping 6 hours. Through this procedure we can suppress some of the noises, but smoothing of the observed dis-

Copy right $(\mathrm{C})$ The Society of Geomagnetism and Earth, Planetary and Space Science (SGEPSS); The Seismological Society of Japan; The Volcanological Society of Japan; The Geodetic Society of Japan; The Japanese Society for Planetary Sciences. placement is unavoidable. The notation "Baseline: A-B" means the coordinates of station $\mathrm{A}$ are subtracted from those of station B. The length components of baselines 9305993060, 93059-960600 and 93060-960600 increased at the time when the earthquake swarm activity in Miyake-jima started. On June 27, all the baselines started to shrink. Only the baseline 93059-960600 was expanding but it turned the trend to shrinking on June 28. This implies that the southern part of Miyake-jima moved southward at the initial stage, and only the southwestern part kept moving for about a day, whereas the other parts started shrinking. After June 28, all the baselines kept shrinking. Changes in the rates of timevariation of baseline vector components were found in association with the eruptions. Superposed on the trends, we can also see short-term changes, which are supposed to be real phenomena and further investigation has been made in the Geographical Survey Institute. Figure 9 shows the displacement of 93059 with respect to 93086 located on the southern tip of the Izu peninsula. In this figure, the coordinates of station were calculated every day using the data covering one day in the Japanese standard time (JST=UT+9 hours). The covered period is from June 17 (JST) to September 3 (JST). With this information, we can estimate the displacement of each station with respect to the southern tip of the Izu peninsula.

\section{Crustal Deformations in Niijima, Kozu-shima and Their Vicinity}

Figures 10 and 11 show the baseline components for the baselines between Niijima (93057) and Kozushima (93058), between Niijima (93057) and Shikine-jima (960597). The data processing procedure and the covering period are the same as in Fig. 9. Respective marks are plotted at the beginning of each day for which processing was made. Both the baselines started to show rapid extension on June 28. The rate of time-variation in the baseline components changes simultaneously with the intense earthquake swarm activity. The total amount of extension of the Niijima to Kozu-shima 


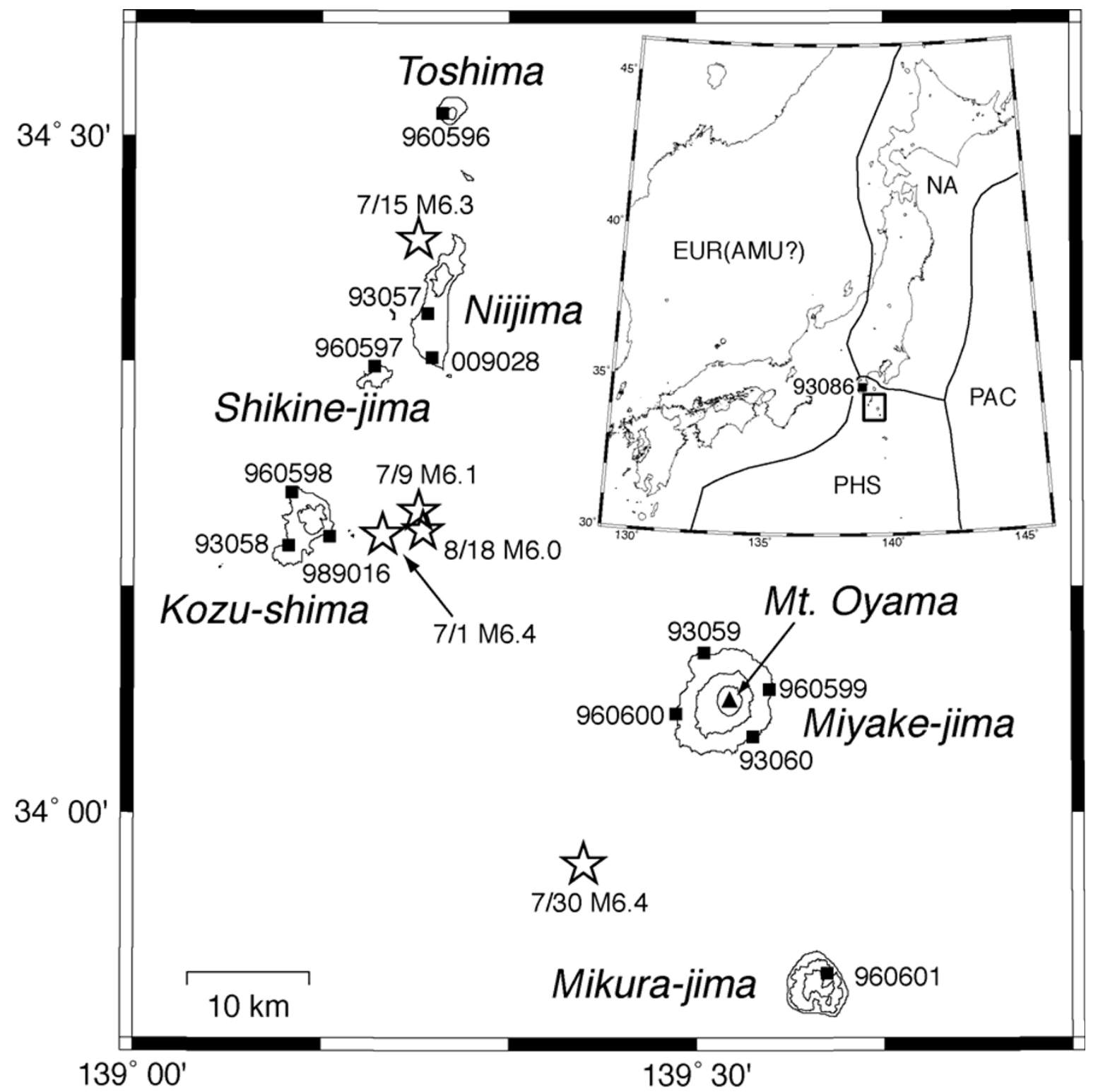

Fig. 1. Location of the area under consideration. EUR: Eurasian plate, AMU: Amur plate, PAC: Pacific plate, PHS: Philippine Sea plate. Squares denote the GPS stations. Star symbols indicate the epicenters of earthquakes of magnitude $\geq 6.0$, according to JMA (Japan Meteorological Agency). 


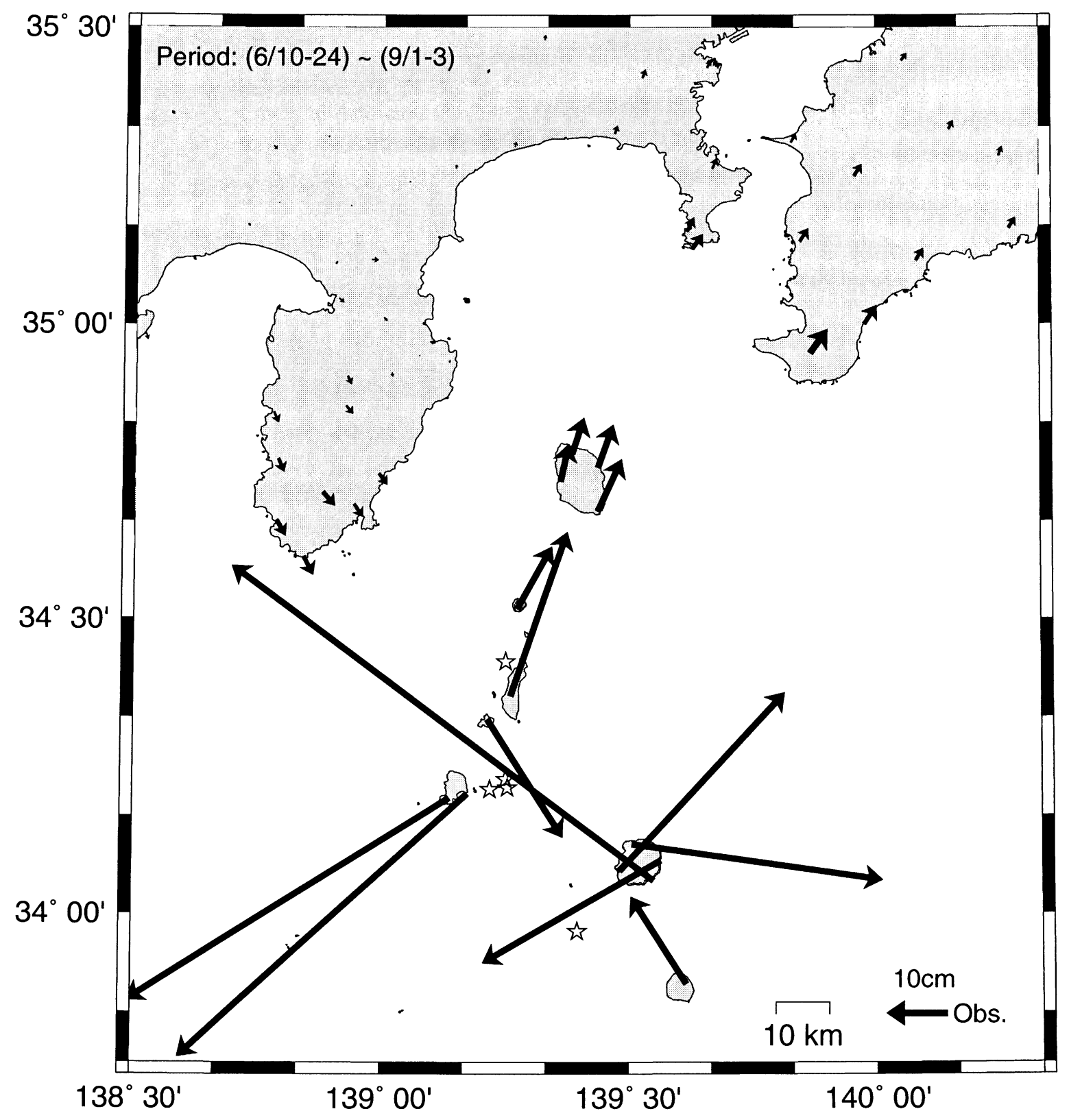

Fig. 2. Displacement vectors at the GPS stations during the period from June 10-24 to September 1-3. In order to remove noises, the coordinates are averaged over 14 days for the reference coordinates before the crsutal activity and over 3 days for the coordinates during the activity. Star symbols denote the epicenters of earthquakes of magnitude $\geq 6.0$, according to JMA. 


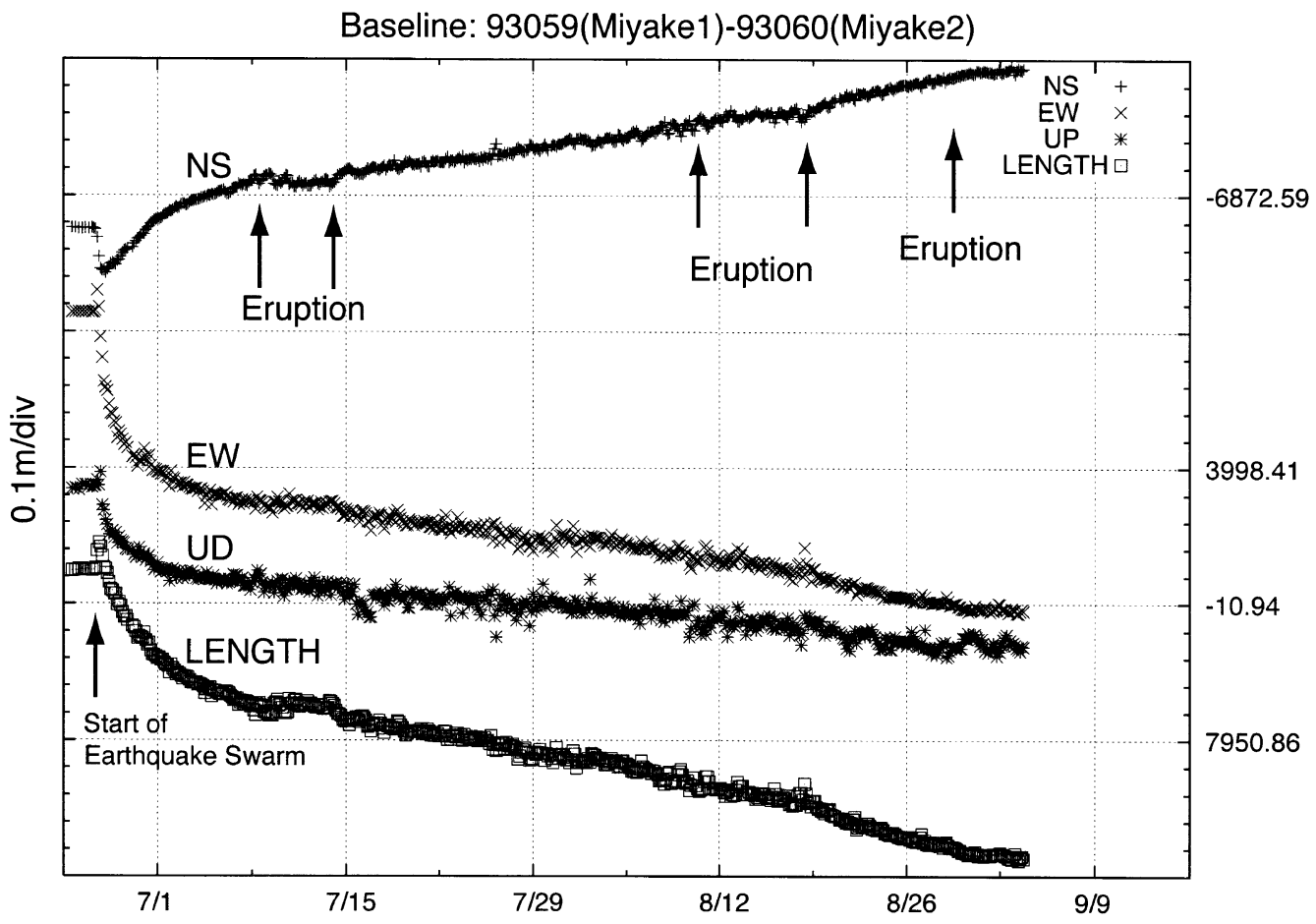

Fig. 3. Temporal changes in the components of baseline 93059-93060. With respect to 93059, the NS component increases when 93060 moves northward. The EW component increases when 93060 moves eastward and the vertical component increases when 93060 moves upward. Arrows denote eruptions of Mt. Oyama.

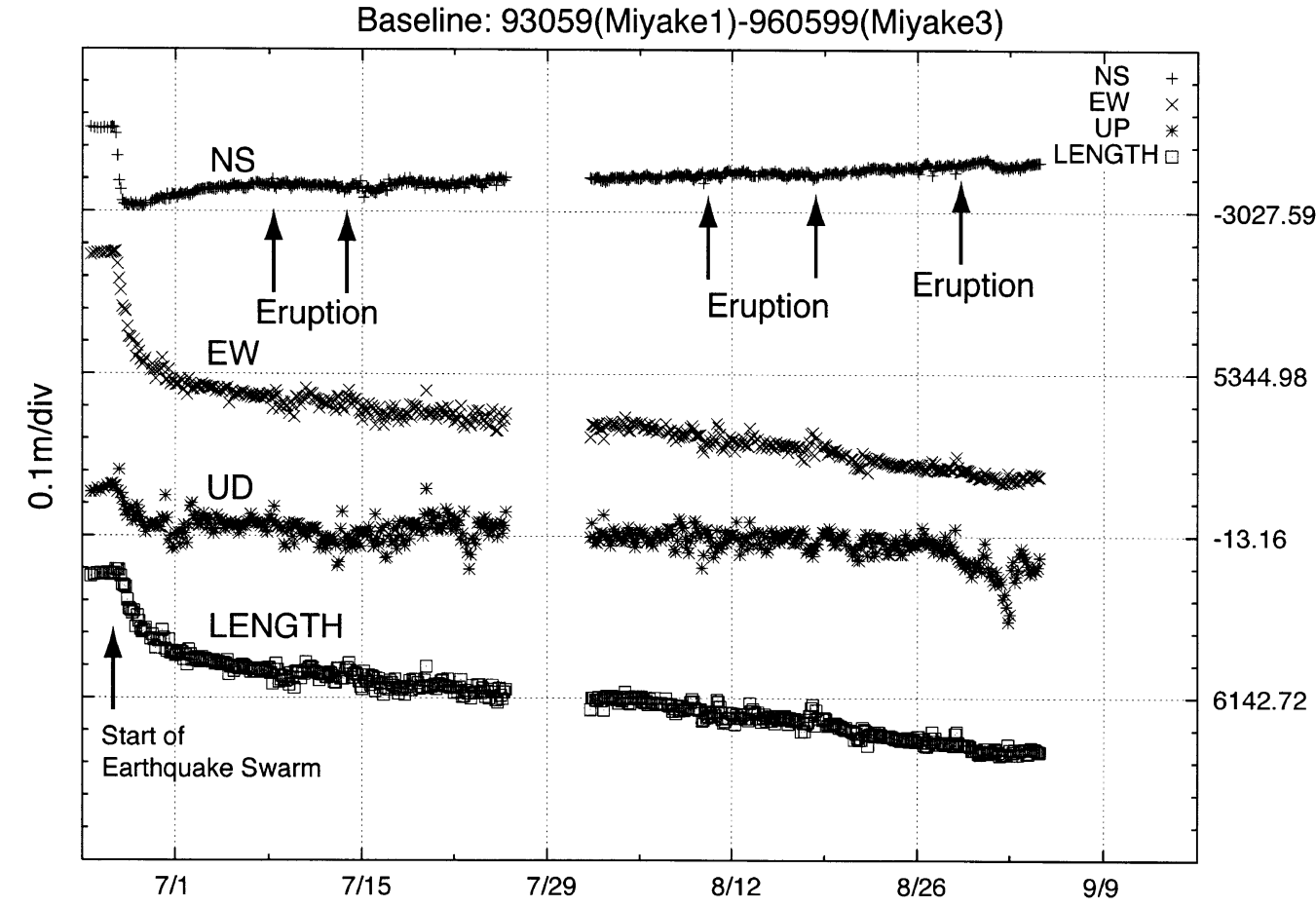

Fig. 4. Temporal changes in the components of baseline 93059-960599. 


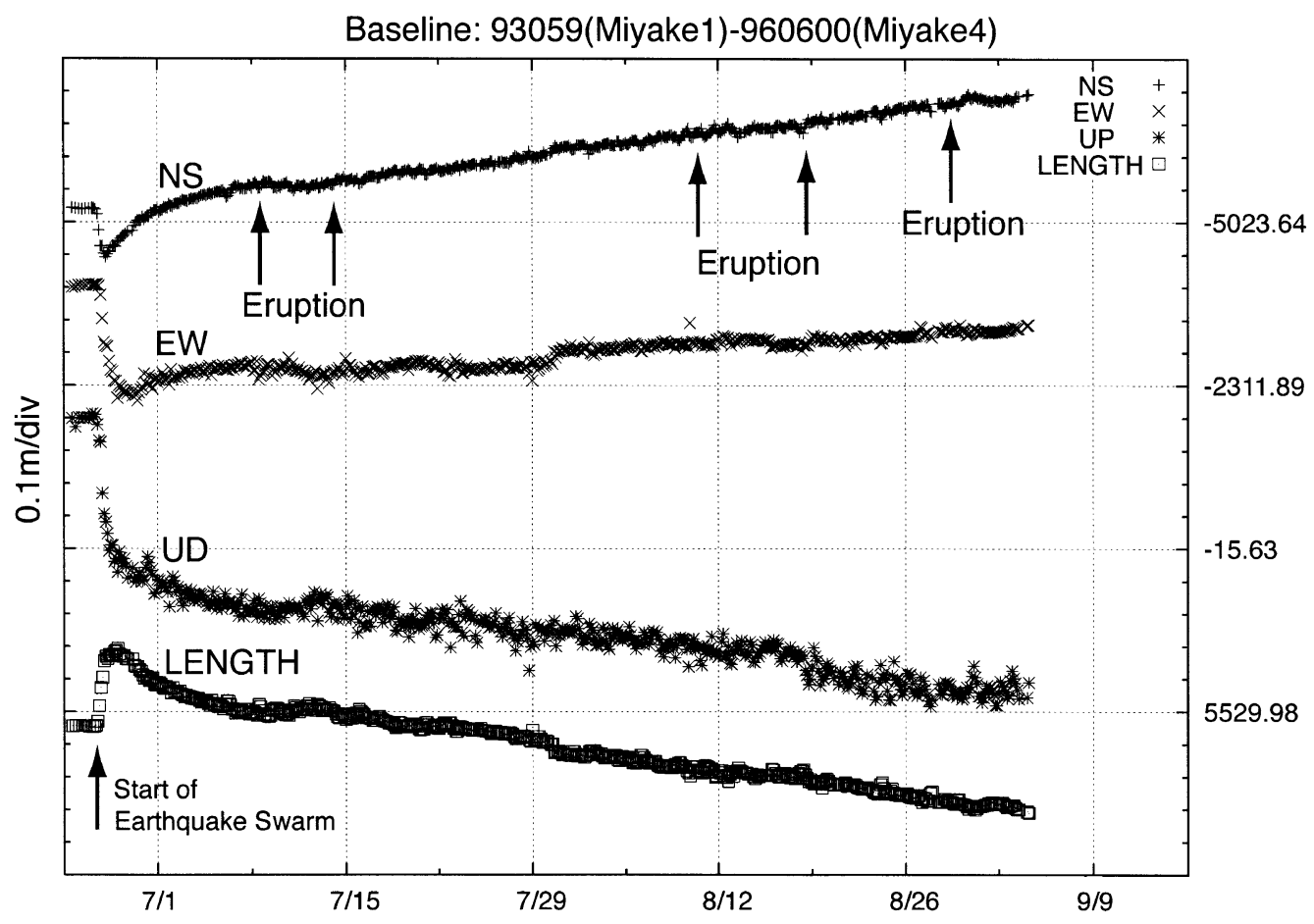

Fig. 5. Temporal changes in the components of baseline 93059-960600.

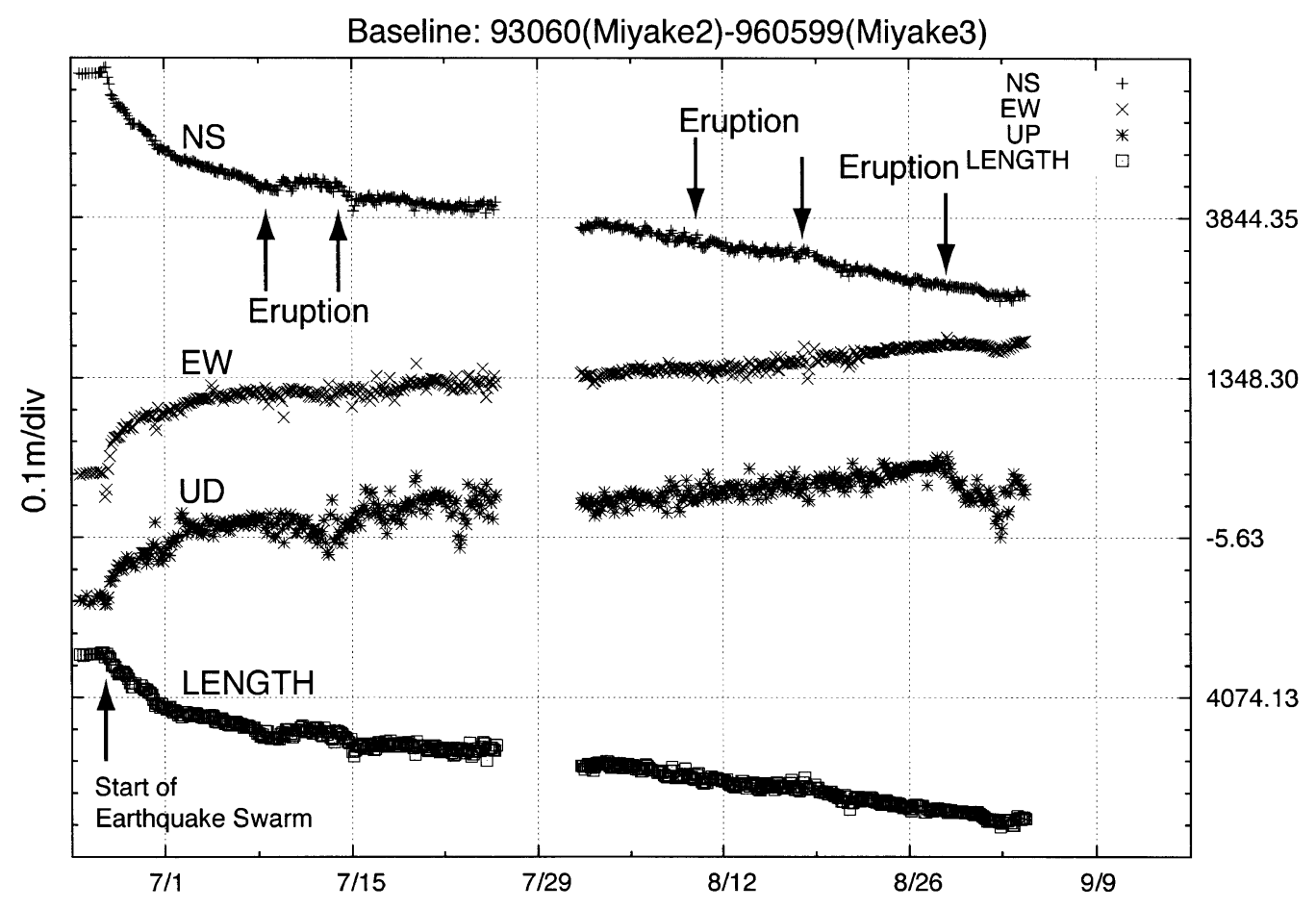

Fig. 6. Temporal changes in the components of baseline 93060-960599. 


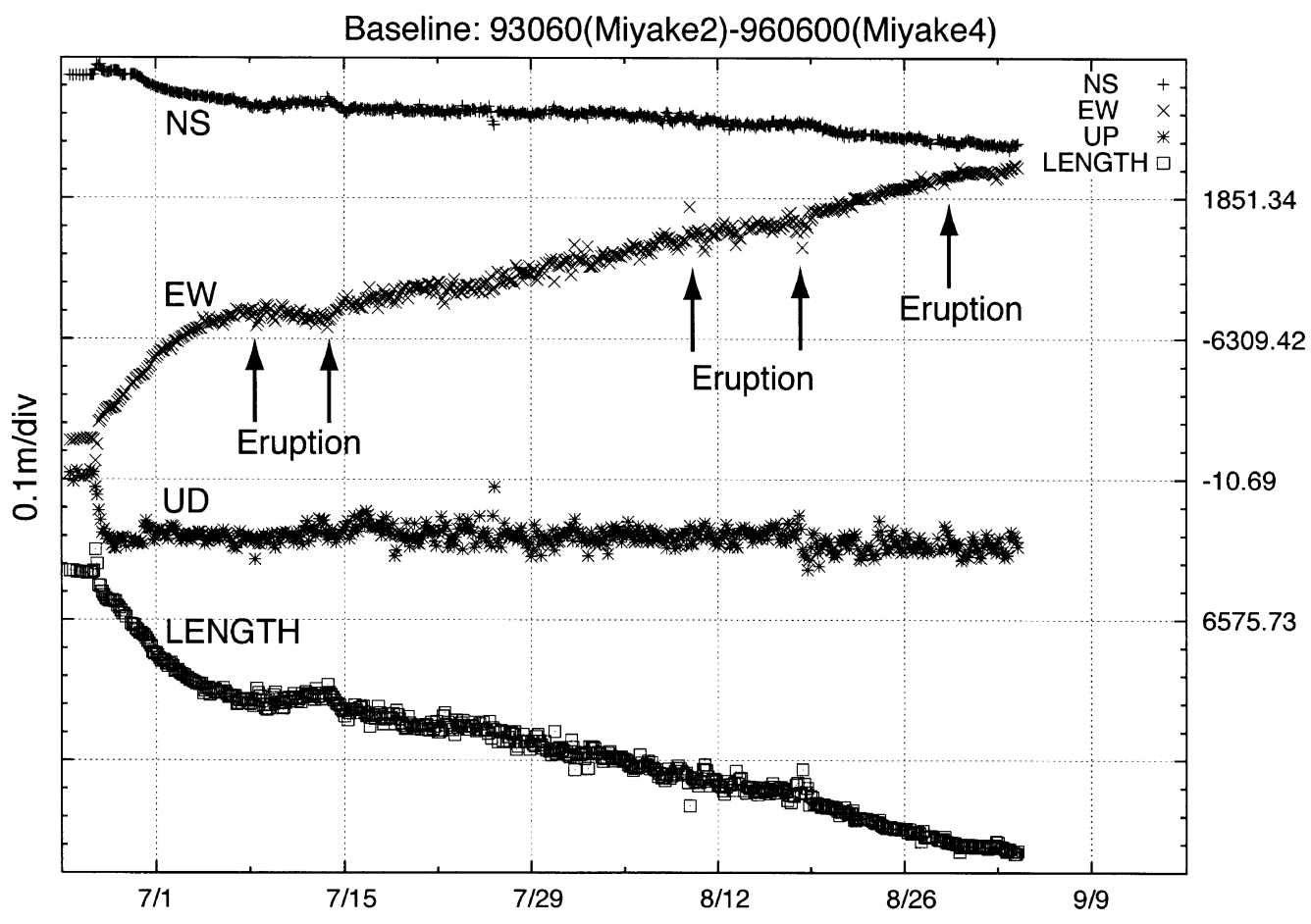

Fig. 7. Temporal changes in the components of baseline 93060-960600.

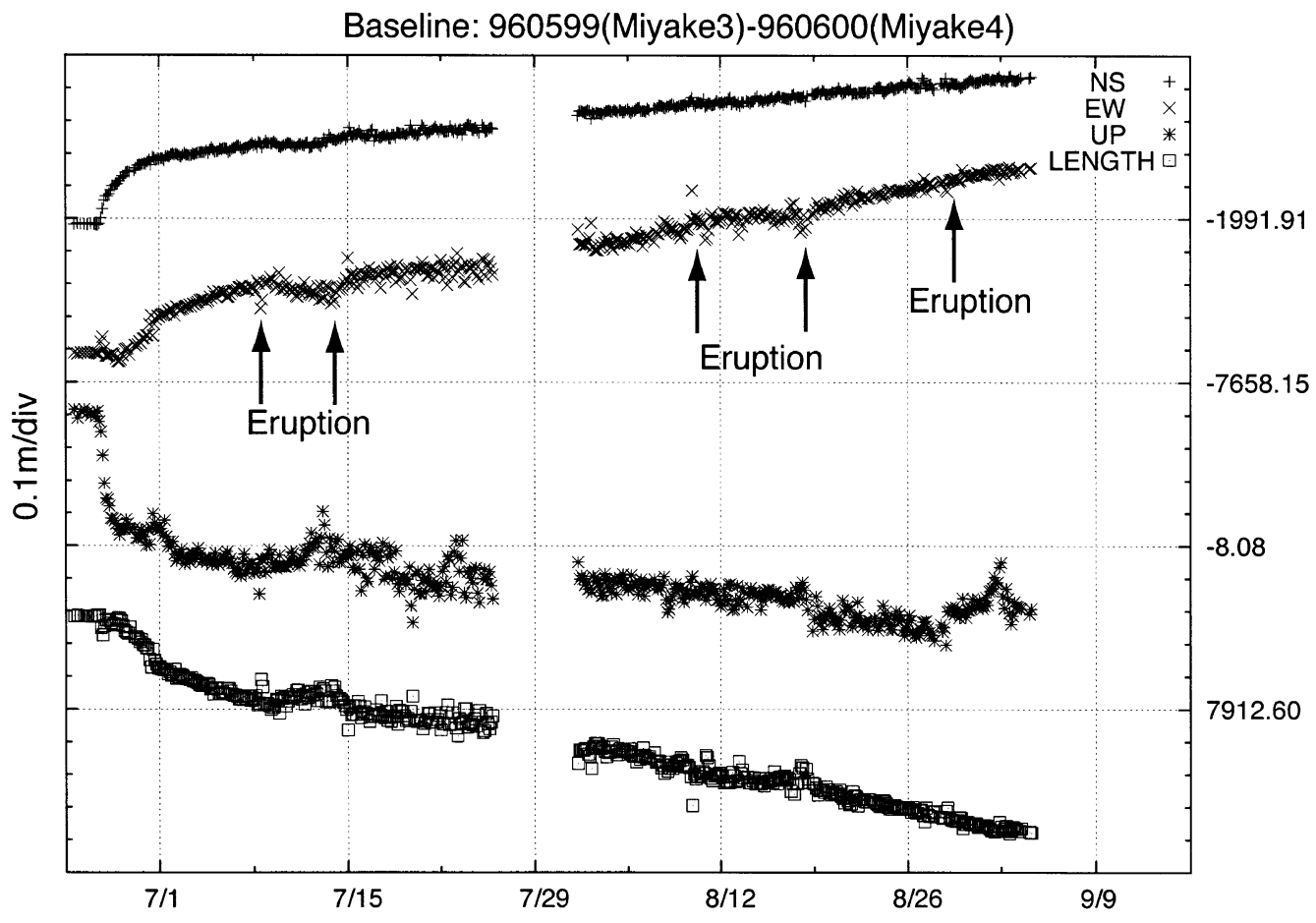

Fig. 8. Temporal changes in the components of baseline 960599-960600. 


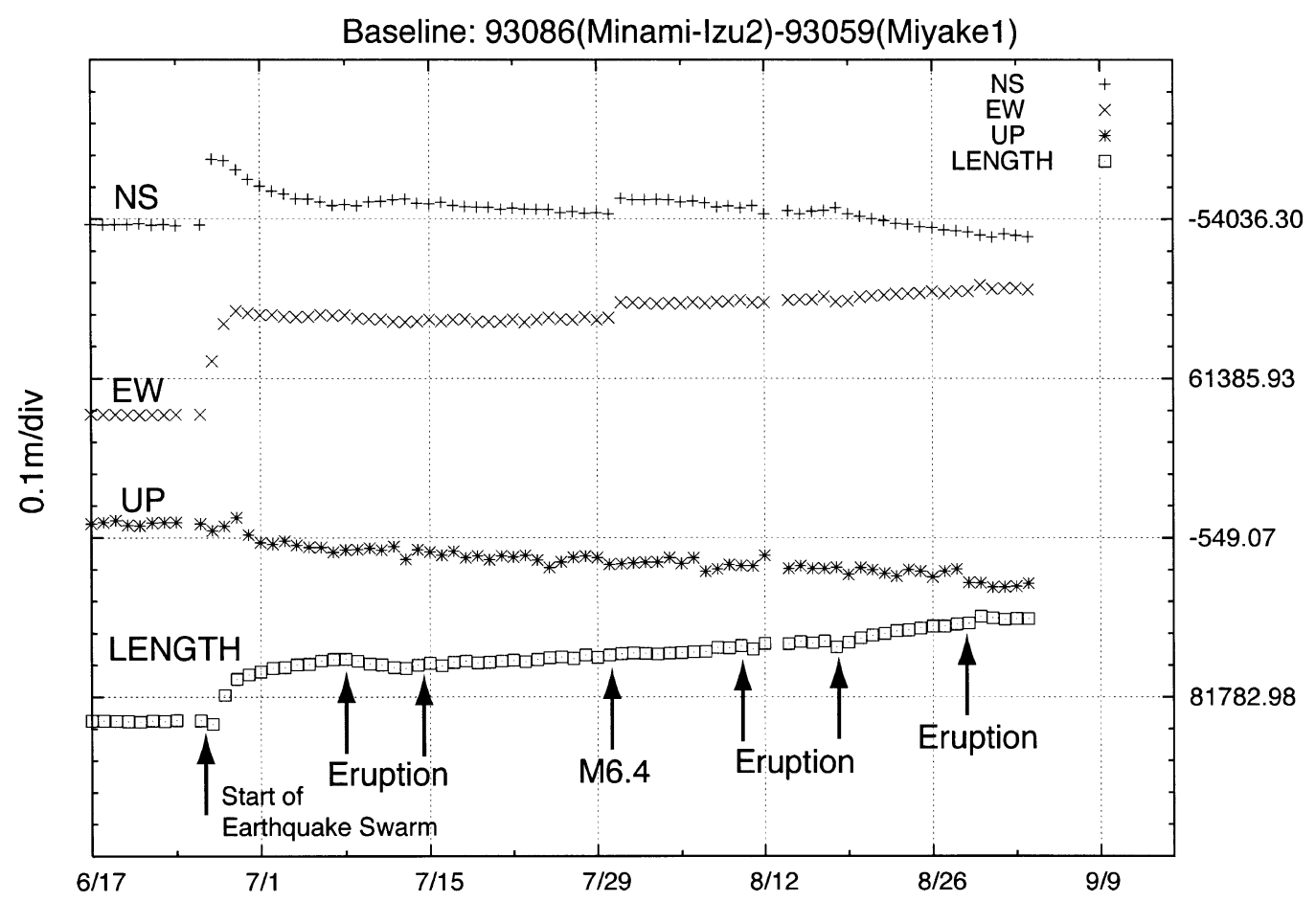

Fig. 9. Temporal changes in the components of baseline 93086-93059. Arrows denote eruptions of Mt. Oyama and the occurrence of an earthquake of magnitude 6.4

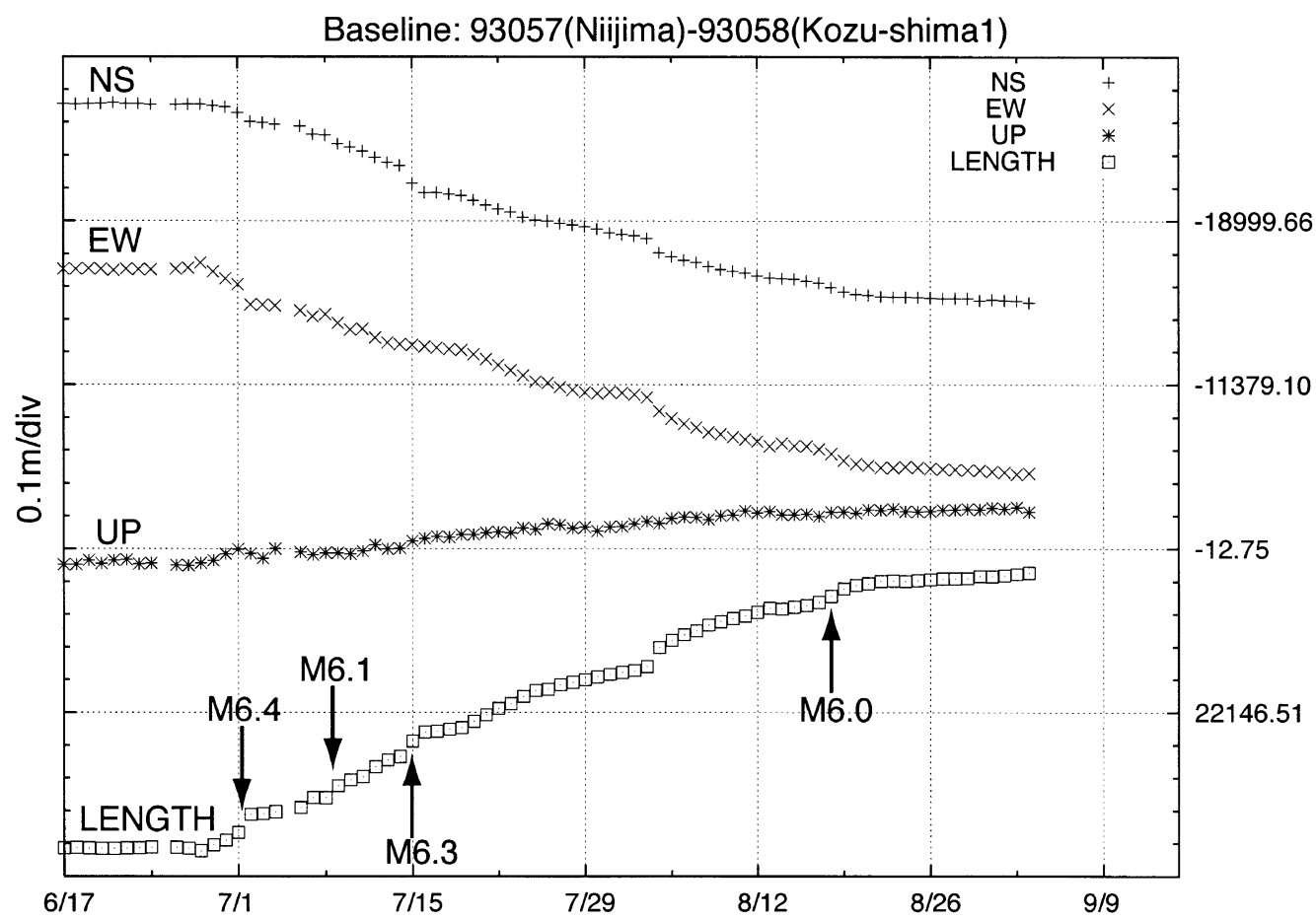

Fig. 10. Temporal changes in the components of baseline 93057-93058. Arrows denote occurrences of earthquakes of magnitude $\geq 6.0$. 


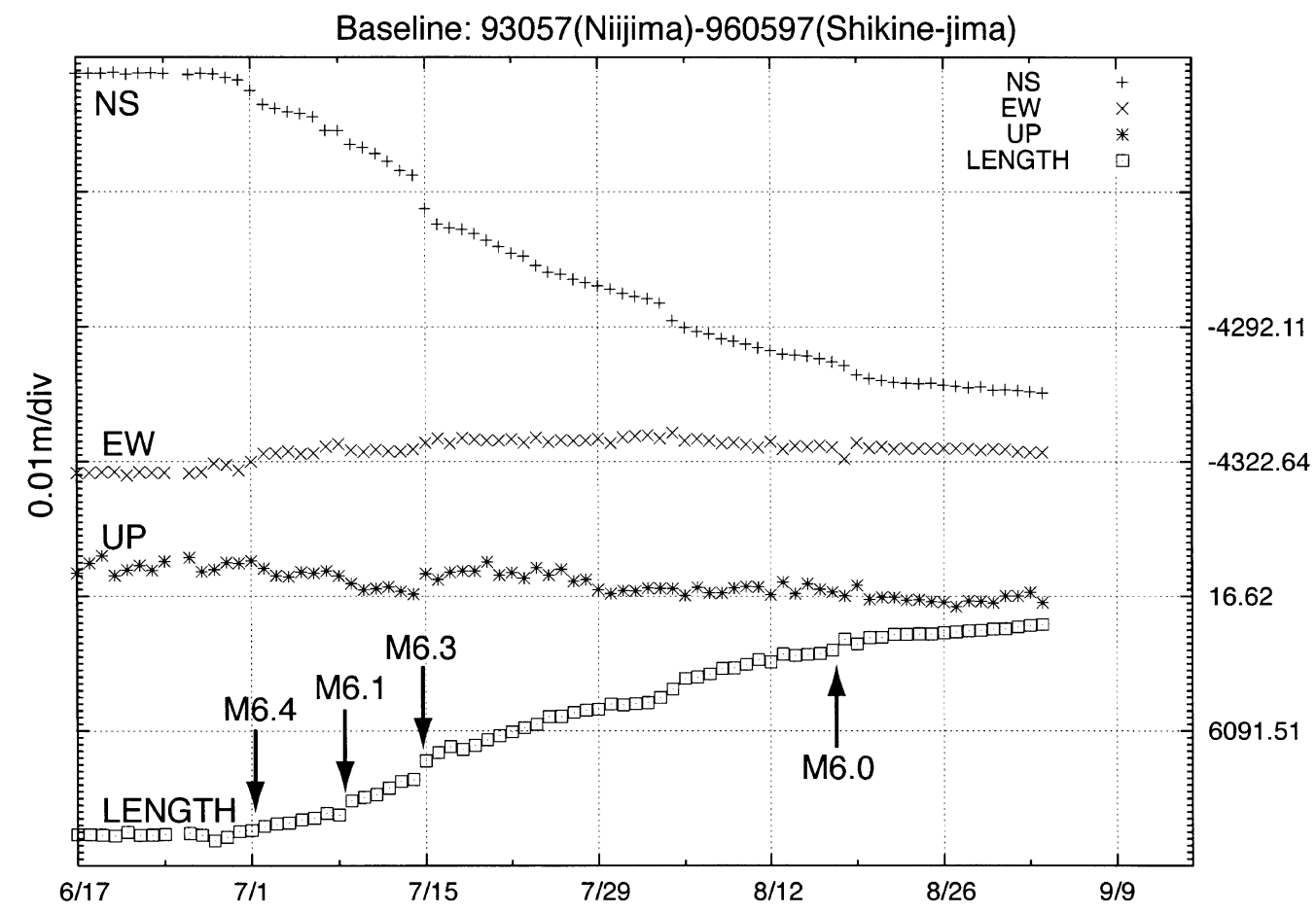

Fig. 11. Temporal changes in the components of baseline 93057-960597.

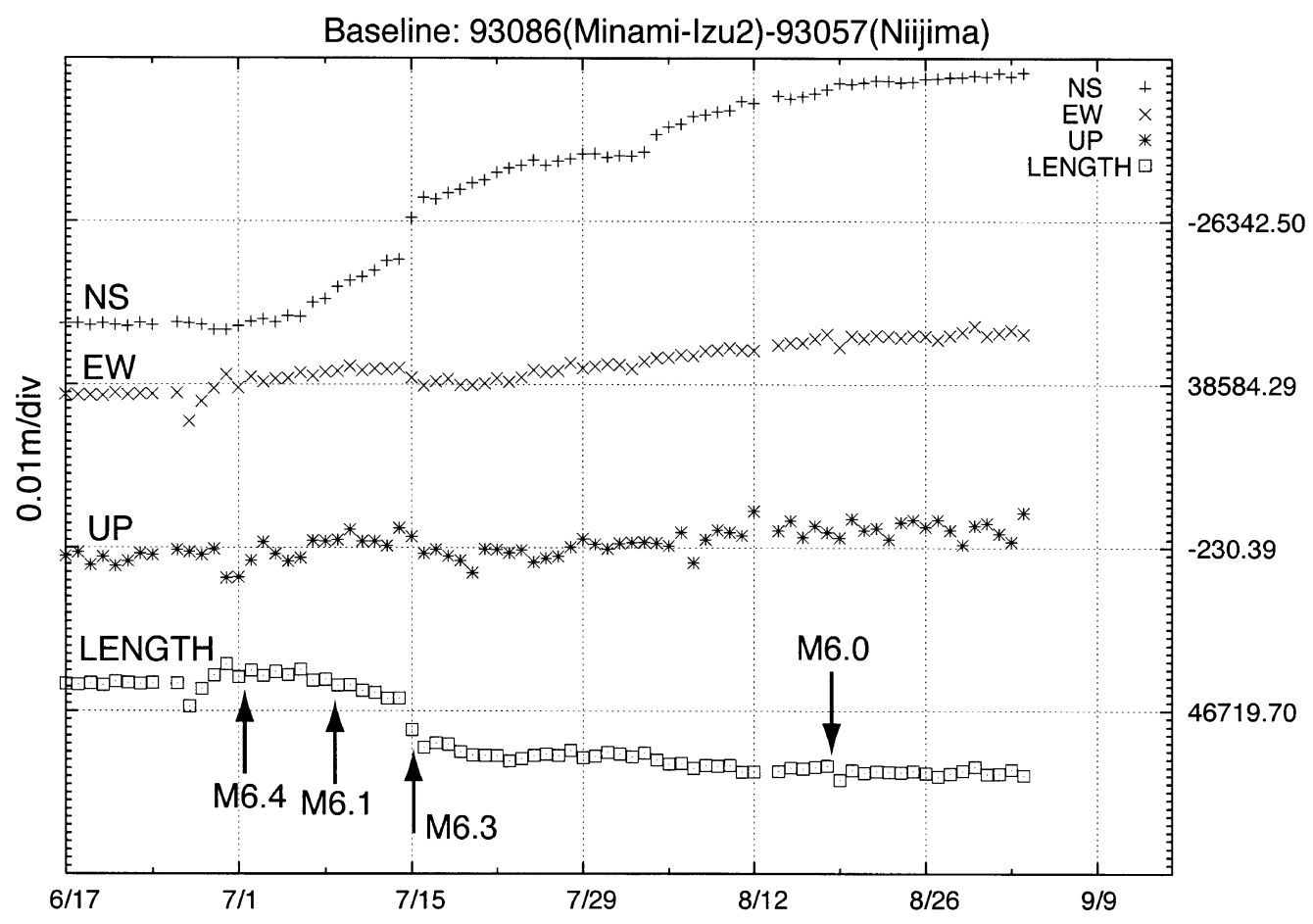

Fig. 12. Temporal changes in the components of baseline 93086-93057. 


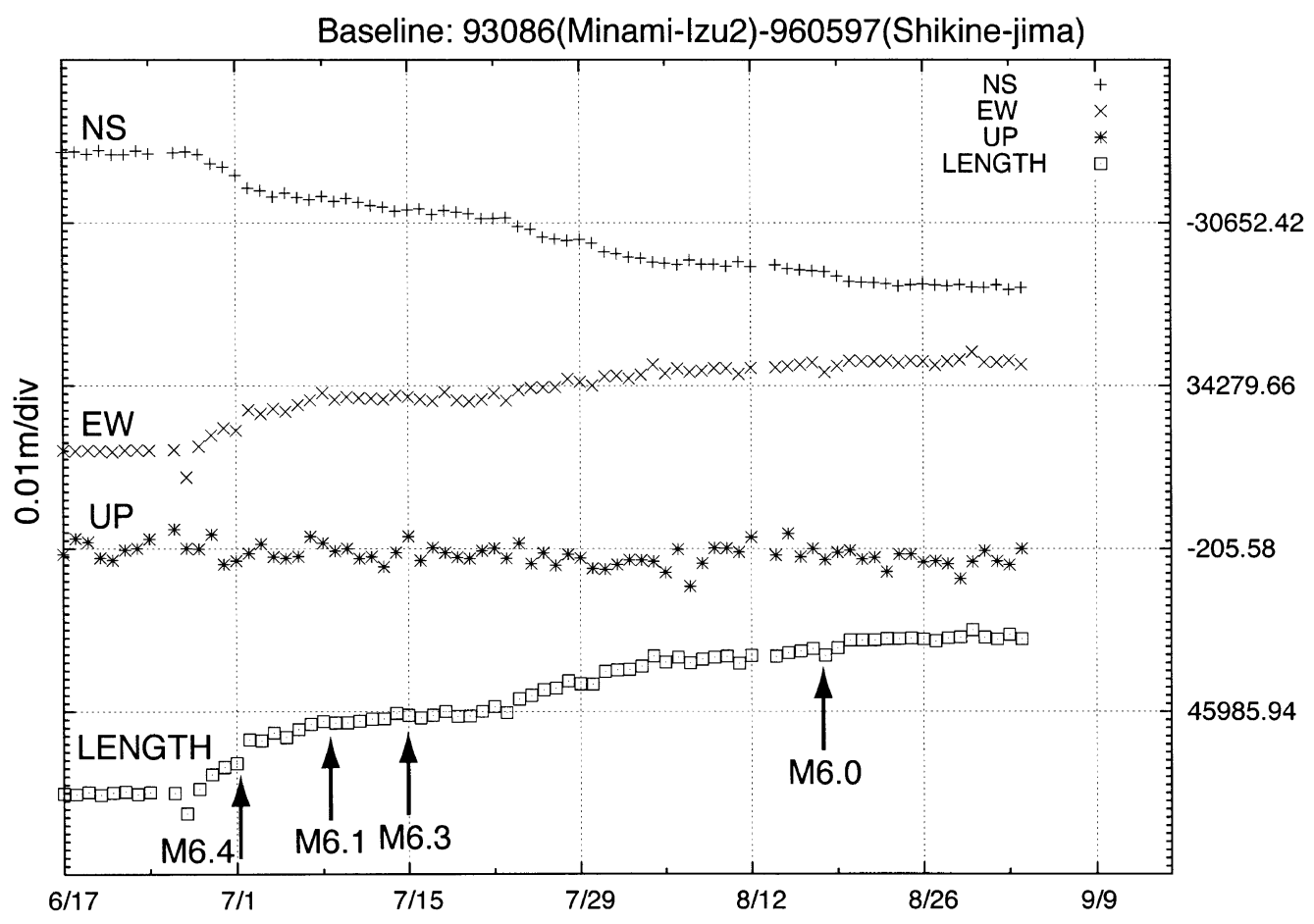

Fig. 13. Temporal changes in the components of baseline 93086-960597.

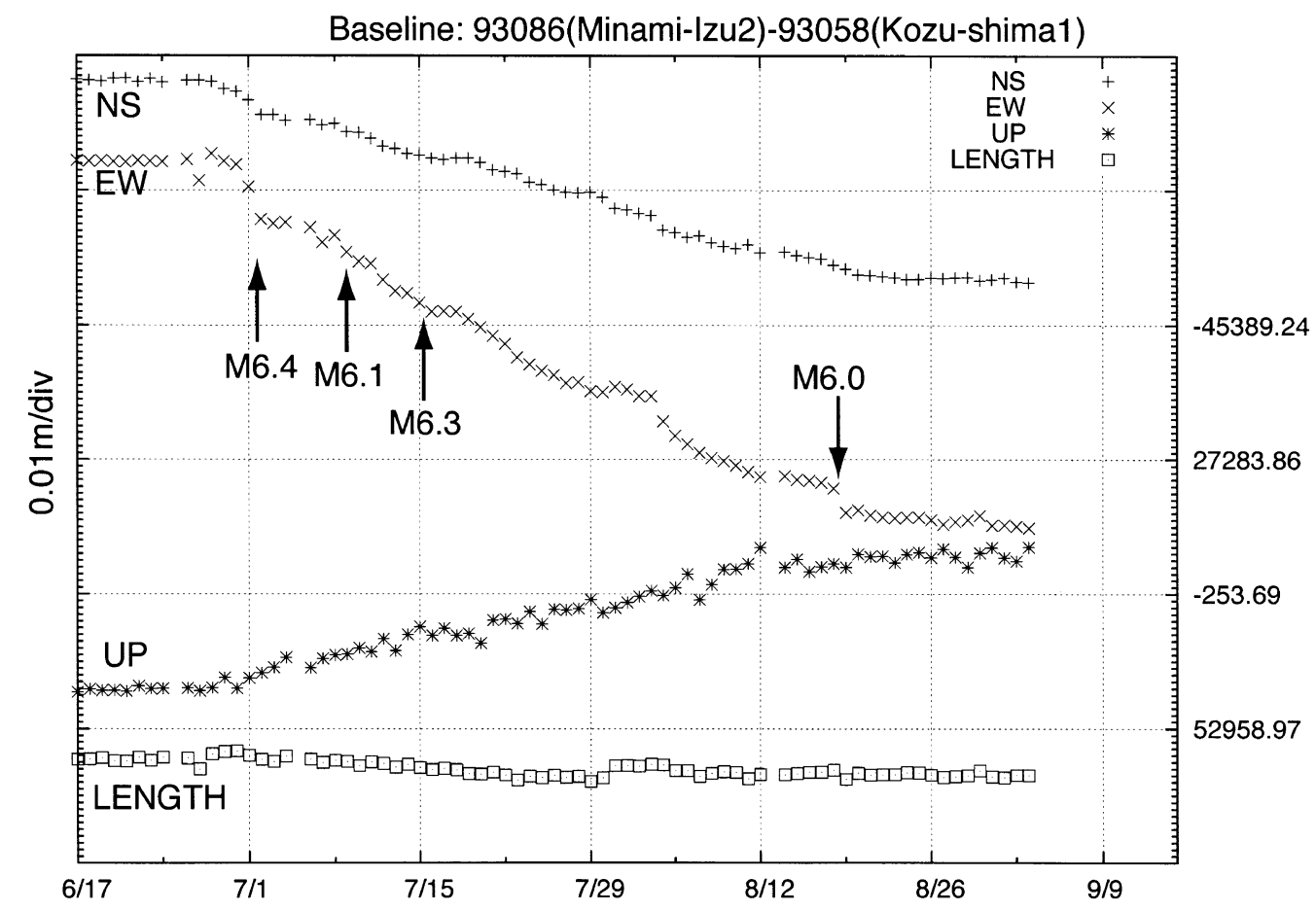

Fig. 14. Temporal changes in the components of baseline 93086-93058. 
baseline, as shown in Fig. 10, is about $0.8 \mathrm{~m}$ since the beginning of the activity. The baseline length is $22 \mathrm{~km}$ and hence the linear strain is 37 microstrain. The linear strain in the baseline connecting Niijima and Shikine-jima during the period is 49 microstrain. The large strain in these baselines, compared to other baselines, suggests that the source causing such deformation is close to the islands and the depth to the source is shallow.

Figures 12 to 14 show the displacements of Niijima, Shikine-jima and Kozu-shima with respect to 93086. From these figures, we can understand that Niijima is moving northward, Shikine-jima southeastward and Kozu-shima southwestward. Upheaval of Kozu-shima and subsidence of Shikine-jima are also significant. These pieces of evidence impose a strong constraint on the source model for the deformations.

Acknowledgments. We thank Kosuke Heki for his valuable comments on the original manuscript.

\section{References}

Miyazaki, S., Y. Hatanaka, T. Sagiya, and T. Tada, The nationwide GPS array as an Earth observation system, Bull. Geograph. Surv. Inst., 44, $11-22,1998$.

M. Kaidzu (e-mail: kaizu@gsi-mc.go.jp), T. Nishimura, M. Murakami, S. Ozawa, T. Sagiya, H. Yarai, and T. Imakiire 